\title{
Wettbewerbsfaktor Wohlfahrtsstaat: eine Annäherung an das vorherrschende Europäische Sozialmodell
}

\author{
Björn Hacker*
}

Häufiger Bezugspunkt sozialwissenschaftlicher Forschung ist der Terminus eines Europäischen Sozialmodells (ESM). Dabei bleibt meist unscharf, was genau unter einem ESM zu verstehen ist. Der Begriff ist schillernd, da er verheißend verwendet wird, und nebulös zugleich, da keine eindeutige Definition existiert, auf welcher Ebene und mit welchen Komponenten ausgestattet das ESM zu verorten ist. Ungeachtet der (richtigen) Auffassung, dass es sich um „ein ebenso unterbestimmtes wie umstrittenes Konzept“ handelt, ${ }^{1}$ ist jedoch eine Annäherung und Klassifizierung möglich. ${ }^{2}$ Hier soll ein Beitrag zu einer Ordnung der zahlreichen vorliegenden Deutungsversuche geleistet werden, indem auf die epistemologischen Besonderheiten unterschiedlicher Forschungsstränge Rücksicht genommen und ihre Perspektiven miteinander verschränkt werden.

Denn in den vorliegenden Arbeiten zum ESM spielen mehrheitlich entweder Aspekte der europäischen Integrationsforschung oder der vergleichenden Wohlfahrtsstaatsforschung eine vorrangige Rolle. Die komparative Analyse differenter Wohlfahrtswelten in der Europäischen Union akzeptiert in Bezug auf das ESM zwar einen historisch gewachsenen, gemeinsamen sozialpolitischen Acquis sowie die Existenz von auf Einzelpolitiken bezogenen Wertvorstellungen. Darüber hinaus fokussiert sie jedoch auf die Singularität von in Clustern organisierten wohlfahrtsstaatlichen Arrangements, die nur durch Pfadbrüche zu überwinden sind. Gegen die Verortung des ESM als modellhafte Idee, deren Verwirklichung an die Gestaltung nationaler Sozialpolitiken gebunden ist, steht seine integrationstheoretische Analyse auf der Gemeinschaftsebene. Darin ist das ESM integraler Bestandteil eines europäischen Lösungswegs für die Überwindung wohlfahrtsstaatlicher Divergenzen und die Annahme gemeinsamer Herausforderungen für alle EU-Staaten. Dieser Weg kann durch einen negativen oder einen positiven Integrationsverlauf befördert werden, führt aber zu unterschiedlichen Ergebnissen, da entweder die ökonomische oder die sozialpolitische Dimension das Bild des ESM prägen.

Diese prinzipiell differenten Ansätze in der Definition des ESM reichen über das wissenschaftliche Spektrum hinaus in die höchst kontrovers geführte politische Diskussion um den künftigen Verlauf der europäischen Integration hinein. Seit der Verankerung des Begriffs in den frühen 1990er Jahren im europapolitischen Diskurs haben sich die verschiedenen politischen Akteure an seiner Deutung, Interpretation und Vereinnahmung versucht. ${ }^{3}$ Welche Perspektive auf die Gestaltung des ESM dabei besonders prägend und einflussreich war und ist, wird durch das vorherrschende theoretische Verständnis von Wohlfahrtsstaatlichkeit und Integrationsprozess deutlich.

* Dr. Björn Hacker, Referent der Internationalen Politikanalyse der Friedrich-Ebert-Stiftung, Berlin.

1 Hans-Wolfgang Platzer: Europäisches Sozialmodell und sozialpolitisches Regieren (in) der EU. Zum integrationspolitischen Kontext und den Perspektiven der Offenen Methode der Koordinierung, in: Stamatia Devetzi/ Hans Wolfgang Platzer (Hrsg.): Offene Methode der Koordinierung und europäisches Sozialmodell. Interdisziplinäre Perspektiven, Stuttgart 2009, S. 83-120, hier S. 85.

2 Vgl. etwa Maria Jepsen/Amparo Serrano Pascual: The European Social Model: An Exercise in Deconstruction, in: Journal of European Social Policy 3/2005, S. 231-245; Wolfram Lamping: Auf dem Weg zu einem postnationalen Sozialstaat? Die Sozialpolitik der Europäischen Union, in: Klaus Schubert/Ursula Bazant/Simon Hegelich (Hrsg.): Europäische Wohlfahrtssysteme. Ein Handbuch, Wiesbaden 2008, S. 595-620.

3 Juho Saari/Kari Välimäki: Seeking a new Balance, in: Jon Kvist/Juho Saari (Hrsg.): The Europeanisation of Social Protection, Bristol 2007, S. 249-266. 


\section{Integrationstheoretische Perspektive: zwischen Marktschaffung und Marktkorrektur}

Die ökonomische Integrationstheorie unterscheidet in der Erweiterung des Wirtschaftsraumes über nationalstaatliche Grenzen hinaus zwischen der positiven und der negativen Integration. Dabei wird die Beseitigung jeder Art von tarifären und nicht-tarifären Handelshemmnissen und Einschränkungen des freien Wettbewerbs zur negativen, die Etablierung gemeinsamer regulativer und wirtschaftspolitischer Kompetenzen zur positiven Integration gezählt. ${ }^{4}$ Während der negative Integrationsprozess vor allem von marktschaffenden Maßnahmen gekennzeichnet ist, die durch die Marktöffnung auf Wettbewerbsintensivierung und Wachstumseffekte abzielen, besteht der positive Integrationsprozess in erster Linie aus marktkorrigierenden politischen Interventionen in das freie Spiel der Marktkräfte.

Für den europäischen Integrationsprozess hat als erster Joseph Weiler auf den Dualismus zwischen supranationalem europäischem Recht und intergouvernementaler europäischer Politik aufmerksam gemacht. ${ }^{5}$ Fritz Scharpf verbindet beide Unterscheidungsebenen: ${ }^{6}$ In der Geschichte der Europäischen Gemeinschaft beziehungsweise Europäischen Union war vor allem die negative Integration ,Nutznießer' des supranationalen Rechts. Dies drückte sich aus in primärrechtliche Verpflichtungen zunächst zur Abschaffung von Binnenzöllen und mengenmäßigen Einfuhrbeschränkungen, später in der Gewährleistung der unbeschränkten Mobilität der vier Faktoren Güter, Arbeit, Kapital und Dienstleistungen. Vertragsverstöße werden durch die Europäische Kommission geahndet und die Reichweite der negativen Integration durch den Europäischen Gerichtshof (EuGH) in seinen Urteilen abgegrenzt. Dagegen ist die positive Integration, die neue gemeinsame Politiken etablieren möchte, in hohem Maße abhängig von Einigungsprozessen im europäischen Mehrebenensystem, besonders durch das Einvernehmen der nationalen Regierungen. Dadurch ergibt sich eine grundlegende, institutionell bedingte „Asymmetrie zwischen den Durchsetzungschancen negativer und positiver Integration in der europäischen Politik“7.

\section{Negativer Integrationsprozess: Sozialpolitik als Produktivitätsfaktor}

Die europäische Integration war bislang vor allem ein Prozess der wirtschaftlichen Vereinigung der europäischen Staaten, in dessen Zentrum mit Binnenmarkt und Währungsunion die Verwirklichung zweier integrationspolitischer Großprojekte stand. Mit dem ESM im Rahmen der marktschaffenden Integration verbindet sich ein neues Paradigma der Staat-MarktBeziehung, das als europäisches Projekt vermittelt wird. ${ }^{8}$ Um die ökonomische Effizienz im gemeinsamen Wirtschafts- und Währungsraum zu steigern und die Preisstabilität in der Eurozone zu wahren, sind die Mitgliedstaaten aufgerufen, staatliche Aktivitäten in der Wirtschaft zugunsten von Privatisierungen und Deregulierung zurückzubauen, tarifvertragliche Regeln zu lockern und die Unternehmensmitbestimmung einzugrenzen, für höhere Flexibilität auf ih-

4 Jan Tinbergen: International Economic Integration, Amsterdam 1954; John Pinder: Positive Integration and Negative Integration - some Problems of Economic Union in the EEC, in: The World Today 3/1968, S. 88-110.

5 Joseph H. H. Weiler: The Community System. The Dual Character of Supranationalism, in: Yearbook of European Law 1981, S. 257-306.

6 Fritz W. Scharpf: Negative and positive Integration in the Political Economy of European Welfare States, in: Gary Marks/Fritz W. Scharpf/Philippe C. Schmitter/Wolfgang Streeck (Hrsg.): Governance in the European Union, London 1996, S. 15-39.

7 Fritz W. Scharpf: Regieren in Europa. Effektiv und demokratisch?, Frankfurt Main 1999, S. 53.

8 Jepsen/Pascual: The European Social Model, 2005; Bernhard Kittel: EMU, EU Enlargement, and the European Social Model: Trends, Challenges, and Questions, Max-Planck-Institut für Gesellschaftsforschung: MPIfG Working Paper 02/1, Köln 2002; Andreas Aust/Sigrid Leitner/Stephan Lessenich: Konjunktur und Krise des Europäischen Sozialmodells. Ein Beitrag zur politischen Präexplantationsdiagnostik, in: Politische Vierteljahresschrift 2/2002, S. 272-301. 
ren Arbeitsmärkten zu sorgen, die Bedingungen für ausländische Kapitalinvestitionen durch Senkung der Lohnnebenkosten und die Reduzierung der Unternehmenssteuersätze zu verbessern sowie die Verschuldung der öffentlichen Haushalte zu reduzieren. Besonders der letztgenannte Punkt hat weitreichende Implikationen für hoch verschuldete Staaten: Zum einen wird der Staat gezwungen, auf nachfrageorientierte Wirtschaftspolitiken weitgehend zu verzichten. Zum anderen stellen die Sozialausgaben in den meisten EU-Staaten den Löwenanteil der gesamten Staatsausgaben dar und sind daher als erstes von möglichen Leistungskürzungen betroffen. Ein im Sinne der negativen Integration verstandenes ESM wirbt mit einem neuen Verständnis von Sozialpolitik als „Produktivitätsfaktor“. ${ }^{9}$ Darin wird die individuelle Verantwortung zum persönlichen Risikomanagement hervorgehoben, die staatliche Garantie sozialer Rechte von der Erfüllung sozialer Pflichten durch den Einzelnen abhängig gemacht und unter den Stichworten Eigenverantwortung, Flexibilisierung und Mobilität das staatliche Wohlfahrtsprogramm zugunsten marktintegrierter Lösungen begrenzt.

Angestrebt wird über die Idee eines hier ökonomisch determinierten ESM eine Art negativer Konvergenzprozess der Sozial-, Arbeitsmarkt- und Beschäftigungspolitiken in der Europäischen Union. Dieser soll die Reibungsverluste der in nationaler Regie verbliebenen Sozial- und Wirtschaftspolitiken mit dem Rechtsbestand des in Binnenmarkt und der Wirtschafts- und Währungsunion (WWU) verwirklichten ökonomischen Integrationsprozesses begrenzen. Daraus soll eine verbesserte Wirtschaftlichkeit resultieren, mit der die Position der Europäischen Union als wirtschaftliche Entität in der globalen Ökonomie optimiert wird. Die Erklärung einer solchen Entwicklung des Integrationsprozesses wird von den Schulen der Europäischen Integrationstheorie unterschiedlich beantwortet. Mit dem Liberalen Intergouvernementalismus lässt sich am ehesten eine Annäherung an ein ESM als Anhängsel der ökonomischen Integration darstellen: Danach bleiben die Nationalstaaten die ,Herren' des Integrationsprozesses ${ }^{10}$ und nutzen ihn als Forum zwischenstaatlicher Verhandlungen für Kooperationen, mit denen die Regierungen besser auf gemeinsame Herausforderungen reagieren können. Bei dieser Art des kooperativen Managements wachsender globaler Interdependenzen sind die Regierungen jedoch nicht frei in ihren Entscheidungen, sondern von in der nationalen politischen Arena ausgebildeten Präferenzordnungen abhängig. Die Konvergenz dieser nationalen Politikpräferenzen führt schließlich zu weiteren Integrationsschritten. ${ }^{11}$ So hat es für einen Prozess der zunehmenden Marktliberalisierung eine einheitliche Position unter den Mitgliedstaaten der Europäischen Union gegeben. Das gleiche gilt für positive marktschaffende Elemente im Rahmen der WWU durch die vergemeinschaftete Geld- und Wechselkurspolitik sowie die strenge Koordinierung der Budgetpolitiken. Dagegen konnte kein gemeinsamer Ansatz gefunden werden für die Etablierung umfassender wirtschaftspolitischer Instrumente und im Bereich der Sozialpolitiken, die folgerichtig in nationalstaatlicher Verantwortung verblieben. ${ }^{12}$

Der Intergouvernementalismus übersieht jedoch auf der einen Seite, dass in einigen Bereichen durchaus sozialpolitische Gemeinschaftsregeln implementiert wurden, die über eine

9 Waltraud Schelkle: Can there be a European Social Model?, in: Erik Oddvar Eriksen/Florian Rödl/Christian Joerges (Hrsg.): Law, Democracy and Solidarity in a Post-National Union: The unsettled Political Order of Europe, London 2008, S. 109-130.

10 Stanley Hoffmann: Obstinate or Obsolete? The Fate of the Nation State and the Case of Western Europe, in: Daedalus 3/1966, S. 862-915.

11 Andrew Moravcsik: Preferences and Power in the European Community: A Liberal Intergouvernmentalist Approach, in: Journal of Common Market Studies 4/1993, S. 473-524.

12 Wilhelm Knelangen: „Sozialstaatswerdung“ Europas? Integrationstheoretische Überlegungen zur Entwicklung der EU-Sozialpolitik, in: Alexandra Baum-Ceisig/Anne Faber (Hrsg.): Soziales Europa? Perspektiven des Wohlfahrtsstaates im Kontext von Europäisierung und Globalisierung, Wiesbaden 2005, S. 20-44, hier S. 35. 
rein marktschaffende Funktion hinausgehen. Auf der anderen Seite kann er nicht erklären, warum sich die Mitgliedstaaten auch im Bereich der angeblich noch souveränen Wirtschaftsund Sozialpolitiken einem Trend zu Deregulierung, Privatisierung, Flexibilisierung und Risikoindividualisierung beugen. Hier kann der Historische Institutionalismus weiterhelfen, der den Blick von einer statischen Analyse von Verhandlungsrunden in den Regierungskonferenzen und Ratszirkeln weglenkt hin zur Betrachtung der zeitlichen Dimension einmal getroffener Entscheidungen. Denn weder sind die Regierungen rein rational agierende Akteure, noch können sie zum Zeitpunkt des Kooperationsabschlusses die weitere Entwicklung des Politikfeldes oder die mögliche Veränderung ihrer eigenen nationalen Präferenzordnung überschauen. In diesen unfreiwillig von den nationalen Regierungen produzierten Kontrolllücken sehen die supranationalen Institutionen die Chance, ihre Kompetenzen im Integrationsraum auszuweiten und ihre Autorität gegenüber den Nationalstaaten zu festigen. ${ }^{13}$

In dieser Sichtweise hätte die Europäische Union aus dem Dilemma ihrer kaum ausgebauten direkten sozialpolitischen Kompetenz eine Tugend gemacht, indem sie die Souveränität der Nationalstaaten in der Wirtschafts-, Beschäftigungs- und Sozialpolitik durch Verfolgung und Einklagung eines mit der Prädominanz der negativen Integration kompatiblen ESM beschneidet, und „,halbsouveräne Wohlfahrtsstaaten“ zurücklässt. ${ }^{14}$ Diese stehen einerseits durch die Wettbewerbslogik des Binnenmarktes, andererseits durch den Verzicht der geld- und wechselkurspolitischen Elemente und die Restriktion ihrer fiskalpolitischen Kapazitäten durch die WWU unter Druck. Sozialpolitik wird in erster Linie als Produktivitätsfaktor für die ökonomische Entwicklung verstanden. Dass der Weg zu einem ESM der negativen Integration bereits beschritten ist, zeigt der europaweite Wettbewerb um möglichst niedrige Steuer-, Sozial-, Lohn- und Beschäftigungsstandards.

\section{Positiver Integrationsprozess: graduelle soziale Flankierung der Wirtschaftsunion}

Im Bereich der positiven Integration ist ein Prozess der kleinen Schritte beobachtbar, der in verschiedenen Phasen unterschiedlich stark wirkende marktkorrigierende Politiken implementiert hat. Besonders der Vertrag von Maastricht kann hier als einschneidendes Ereignis hervorgehoben werden. Mit ihm fand eine Aufwertung der bis dahin in erster Linie als Beiwerk der Binnenmarktintegration zu verstehenden arbeits- und gleichstellungsrechtlichen Regulierung statt. Die Aufgabe des Einstimmigkeitsprinzips bei Ratsentscheidungen in einigen sozialpolitischen Themenfeldern hat dabei geholfen, die von Scharpf 1985 beschriebene „Politikverflechtungsfalle“15 in der EU-Sozialpolitik zu überwinden. Ferner hat der Bedeutungs- und Machtzuwachs für die Beziehungen zwischen Arbeitgebern und Arbeitnehmern auf verschiedenen Ebenen des europäischen Integrationsfeldes zum Ausgang aus der von Wolfgang Streeck konstatierten ,korporatistischen Entscheidungslücke“16 geführt. Darüber

13 Paul Pierson: The Path to European Integration. A Historical Institutionalist Analysis, in: Comparative Political Studies 2/1996, S. 123-163; Daniel Wincott: Institutional Interaction and European Integration: Towards an Everyday Critique of Liberal Intergouvernementalism, in: Journal of Common Market Studies 4/1995, S. 597609.

14 Paul Pierson/Stephan Leibfried: Semisouvereign Welfare States: Social Policy in a Multitired Europe, in: Stephan Leibfried/Paul Pierson (Hrsg.): European social policy. Between fragmentation and integration, Washington 1995, S. 43-77.

15 Fritz W. Scharpf: Die Politikverflechtungsfalle: Europäische Integration und Deutscher Föderalismus im Vergleich, in: Politische Vierteljahresschrift 4/1985, S. 323-356.

16 Wolfgang Streeck: Politikverflechtung und Entscheidungslücke. Zum Verhältnis von zwischenstaatlichen Beziehungen und sozialen Interessen im europäischen Binnenmarkt, in: Fritz W. Scharpf/Karlheinz Bentele/ Bernd Reissert/Ronald Schettkat (Hrsg.): Die Reformfähigkeit von Industriegesellschaften, Frankfurt Main 1995, S. 101-130. 
hinausgehend konnte durch die EU-Gleichstellungspolitik, die in jüngster Zeit zunehmend von einer im Fokus erweiterten Antidiskriminierungspolitik ${ }^{17}$ ergänzt wird, abseits der verhandelten Regulierung ein eigenes primärrechtliches Aktionsfeld der sozialen Dimension verankert werden, dessen Umfang und Reichweite sich vor allem durch die expansive Rechtsprechung des EuGH konstituiert hat. Mit der Europäischen Beschäftigungsstrategie, dem Makroökonomischen Dialog und der Offenen Methode der Koordinierung (OMK) im Bereich der sozialen Sicherheit konnte ein weiterer - allerdings voluntaristischer - Schritt zur Stärkung der positiven Integration getan werden.

Ein vor diesem Hintergrund verstandenes ESM identifiziert einen positiven Integrationsprozess, der durch seine marktkorrigierende Funktion zur Herausbildung einer sozialen Dimension der Europäischen Union führt und deren ökonomische Grundlagen ergänzt. Als Zielbeschreibung dient die Gleichwertigkeit der wirtschaftlichen und der sozialpolitischen Integration im Sinne zweier Pendants. Betont wird dabei die Notwendigkeit der sozialen Flankierung des Binnenmarkts und der Währungsunion: Um deren Vorteile voll ausschöpfen zu können, könne nicht allein auf Wettbewerb und Liberalisierung gesetzt werden. Sozialpolitik dürfe nicht als störend für den Prozess der Marktschaffung oder zweckentfremdet und wichtiger Instrumente beraubt als reiner Produktivitätsfaktor empfunden werden. Denn in dem Maße, wie Sozialpolitik auf nationaler Ebene beschränkt und gekürzt wird, aber nicht auf supranationaler Ebene der ökonomischen Integration nachwächst, zeigen sich Probleme, die zuvor von den Sozialpolitiken behandelt und gelöst werden konnten, in neuem Ausmaß, unter Umständen auch an anderen Stellen in der Gesellschaft. Der soziale Frieden aber ist die unbedingte Grundvoraussetzung für die Generierung von wirtschaftlichem Fortschritt. Iain Begg hat dieses Phänomen mit „the costs of non-social policy“ bezeichnet. ${ }^{18}$

Die Vision eines ESM der positiven Integration erklärt sich in erster Linie durch ein funktionales Integrationsverständnis. Danach haben die Mitgliedstaaten der Union in den 1950er Jahren einen Prozess der technischen Kooperation in Wirtschaftsfragen begonnen, der sich durch Spill-over-Prozesse über die Zeit in den politischen Raum ausgedehnt hat. ${ }^{19}$ Dabei spielen die gesellschaftlichen Akteure durch Ausübung von Druck zu einer vertieften Integration (,political spill-over'), von der sie sich Vorteile versprechen, eine wichtige Rolle. Auch die supranationalen Institutionen werden aktiv, indem sie diesen Druck instrumentalisieren, um ihren eigenen Einflussbereich auszubauen (,cultivated spill-over'). Der Neo-Funktionalismus identifiziert seit der Einheitlichen Europäischen Akte (EEA) beide Prozesse in der Europäischen Gemeinschaft durch den Wunsch der Arbeitgeber nach einer Ausweitung des gemeinsamen Wirtschaftsraumes und den Forderungen der Gewerkschaften zum Ausbau der sozialen Dimension sowie durch die Ausweitung der supranationalen Zuständigkeiten durch Kommission und EuGH. Dies mündete in einem ,functional spill-over', in dem die negative Integrationsdynamik durch Aspekte der positiven Integration erweitert wurde. ${ }^{20}$ Ergänzt um die vom Historischen Institutionalismus erkannten, von den Regierun-

17 Vgl. aber den berechtigten Einwand, dass Antidiskriminierungspolitik ,kein Bestandteil und auch kein Substitut einer marktkorrigierenden Politik" ist. Siehe Martin Höpner/Armin Schäfer: Grenzen der Integration - wie die Intensivierung der Wirtschaftsintegration zur Gefahr für die politische Integration wird, in: integration 1/2010, S. 3-20, hier S. 17.

18 Iain Begg: Rethinking the Social Dimension of the EU: The Costs of Non-Social Policy, in: Alexandra BaumCeisig/Anne Faber (Hrsg.): Soziales Europa? Perspektiven des Wohlfahrtsstaates im Kontext von Europäisierung und Globalisierung, Wiesbaden 2005, S. 293-312.

19 Ernst B. Haas: The Uniting of Europe. Political, social, and economic Forces 1950 - 1957, London 1958.

20 Jeppe Tranholm-Mikkelsen: Neo-functionalism: Obstinate or Obsolete? A Reappraisal in the Light of the New Dynamism of the EC, in: Millennium 1/1991, S. 1-22; Philippe C. Schmitter: Neo-Neofunctionalism, in: Antje Wiener/Thomas Diez (Hrsg.): European Integration Theory, Oxford 2004, S. 45-74. 
gen nicht antizipierten und intendierten Konsequenzen im Bereich der sozialpolitischen Gesetzgebung steht das Gerüst eines Erklärungsansatzes für das ESM der positiven Integration.

Dabei darf jedoch nicht übersehen werden, dass die Nationalstaaten den Prozess der positiven Integration nur bis zu einem bestimmten Punkt zugelassen haben. So war der sozialpolitische Integrationsfortschritt von der EEA zum Vertrag von Maastricht zwar substanziell, danach ist jedoch ein Rückgang der Dynamik zu verzeichnen: Abseits beachtlicher Fortschritte im normativen Bereich, etwa durch die Rechtsverbindlichkeit der Grundrechtecharta, die Einführung einer horizontalen Sozialklausel und die vertraglich fixierte sozialpolitische Zielbestimmung der Europäischen Union, bleibt es hinsichtlich der institutionellen Ausgestaltung und den politischen Kompetenzen bei einem „konstitutionellen Minimalismus“21. Die Weiterentwicklung der positiven Integration mit dem Vertrag von Amsterdam war nur graduell, zum darauf folgenden Vertrag von Nizza minimal und mit dem Vertrag von Lissabon wird nur der status quo ante fortgeschrieben. ${ }^{22}$ Europäische Sozialpolitik findet ansatzweise allein im Bereich der sozialregulativen, nicht jedoch in der als Konstituante des Wohlfahrtsstaats ungleich wichtigeren redistributiven Sozialpolitik statt. Zudem besteht eine eindeutige Tendenz eines immer stärkeren Vorstoßes in den Bereich der freiwilligen, unverbindlichen Politikkoordinierung, mit der die rechtlich verbindliche sozialpolitische Gesetzgebung zunehmend abgelöst wird. ${ }^{23}$

Ein ESM der positiven Integration bleibt angesichts des aktuellen Integrationsstands eine vage Zukunftsvision, die eher mit föderalistischen Wünschen an die Errichtung einer politischen Union zu vereinbaren ist, als mit der realen Binnenmarkt- und Währungsraumintegration. Dennoch ist ein gewisser gemeinsamer sozialpolitischer Acquis ein Faktum, der in einigen wenigen Bereichen über eine Funktion als ergänzender Wegbereiter des Wettbewerbs hinausgeht. Man mag diese Errungenschaften als punktuelle Schnittstellenharmonisierung ${ }^{24}$ abtun, doch scheint ein Kernbestand des ESM aus sozialen Werten und Prinzipien bereits heute vorhanden zu sein. Diese sind teilweise primärrechtlich verankert und bestehen aus einem Set an arbeitsrechtlichen sowie diversen weiteren Modi der Regulierung durch Kollektivverhandlungen und sozialpolitischer Koordinierung.

\section{Komparativ-wohlfahrtsstaatstheoretische Perspektive: zwischen politischem Erbe und neuem Design}

Unter Berücksichtigung des Grades an Dekommodifizierung - das heißt der individuellen Unabhängigkeit vom Markt durch soziale Absicherung - sowie der Stratifizierung einer Gesellschaft - das heißt der Messung von Persistenz oder Auflösung von Statusunterschieden durch soziale Umverteilung - konstruierte Gøsta Esping-Andersen drei Wohlfahrtsstaatsregime, in denen die Bedeutung der drei zentralen Versorgungsinstitutionen mit sozialer Sicherheit - Staat, Markt und Familie - unterschiedlich ausgeprägt sind und denen sich Staatengruppen zuordnen lassen. ${ }^{25}$ Dies sind:

21 Hans-Wolfgang Platzer: Konstitutioneller Minimalismus: die EU-Sozialpolitik in den Vertragsreformen von Nizza bis Lissabon, in: integration 1/2009, S. 33-49.

22 Ebenda; Platzer: Europäisches Sozialmodell, 2009, S. 96.

23 Simone Leiber/Armin Schäfer: Der doppelte Voluntarismus in der EU-Sozial- und Beschäftigungspolitik, in: Ingeborg Tömmel (Hrsg.): Die Europäische Union. Governance und Policy-Making, Wiesbaden 2008, S. 116135.

24 Vgl. etwa Fritz W. Scharpf: Community and autonomy: Multi-level policy-making in the European Union, in: Journal of European Public Policy 2/1994, S. 219-242.

25 Gøsta Esping-Andersen: The three worlds of welfare capitalism, Princeton 1990; Gøsta Esping-Andersen: Social foundations of postindustrial economies, New York 1999. 
(1) Im liberalen Wohlfahrtsstaat müssen die Bürgerinnen und Bürger ihre soziale Sicherheit in hohem Maße durch individuelle Vorsorge privat organisieren, in dem der Dekommodifizierungsgrad und die Einkommensumverteilung gering sind und über eine Grundversorgung hinaus lediglich ein Minimum an sozialer Fürsorge für die Bedürftigsten durch den Staat gewährleistet wird. Länderbeispiele für den liberalen Wohlfahrtsstaat in Europa sind Großbritannien und Irland.

(2) Der konservative Wohlfahrtsstaat verbindet die soziale Sicherheit eng mit der Erwerbstätigkeit und gewährleistet durch starke korporatistische und etatistische Elemente einen mittleren Grad an Dekommodifizierung. Der Markt spielt als Bereitsteller sozialer Leistungen nur eine sehr geringe Rolle, dafür wird die tradierte soziale Sicherung durch die Familie gefördert. Da die Höhe staatlicher Sozialleistungen stark an das Einkommen gebunden ist, wirkt der konservative Wohlfahrtsstaat nur in geringem Maße umverteilend; Statusunterschiede innerhalb der Gesellschaft bleiben erhalten. Deutschland, Frankreich und Österreich sind Vertreter der konservativen Wohlfahrtswelt.

(3) Der sozialdemokratische Wohlfahrtsstaat vereint eine universalistische Abdeckung der Bevölkerung durch ein staatliches Netz sozialer Sicherheit mit einer hohen Unabhängigkeit des Individuums vom Marktgeschehen und von der Familie. Statusunterschiede durch Klasse und Beruf werden durch weitreichende Umverteilungselemente beseitigt. Schweden, Dänemark und Finnland stehen für dieses Wohlfahrtsstaatsmodell.

Die Konzentration auf die Aspekte Dekommodifizierung und Stratifizierung wurde an Esping-Andersens Ansatz häufig kritisiert und zahlreiche Erweiterungen, Neuberechnungen und veränderte Schwerpunktsetzungen vorgenommen. ${ }^{26}$

Eine eigene Kategorie (4) rudimentärer Wohlfahrtsstaaten bilden jene Länder, in denen ähnlich wie in der konservativen Wohlfahrtswelt - korporatistische und familiäre Strukturen eine starke Rolle spielen, in denen aber zusätzlich die Kirche einen starken Einfluss auf die soziale Sicherung ausübt. Diese Staatengruppe - Vertreter sind unter anderem Spanien, Portugal und Griechenland - schwankt zwischen einem hoch ausgebauten, teilweise universellen Netz sozialer Sicherheit und einer kaum gewährleisteten Versorgung durch den Staat außerhalb der Erwerbstätigkeit. Typisch sind Manipulationen der Sozialsysteme durch Korruption und politischen Klientelismus. ${ }^{27}$

Auch wenn sie durch ihre gemeinsame Vergangenheit einige Gemeinsamkeiten aufweisen, scheint kein eigener (5) postsozialistischer Wohlfahrtsstaatstypus für die zehn 2004 und 2007 zur Europäischen Union gestoßenen mittel- und osteuropäischen Länder zu existieren. Während nach der politischen Wende zunächst einheitlich Tendenzen einer universalistischen Gesundheitsversorgung in Kombination mit bismarckschen Renten- und Arbeitslosenversicherungen beobachtbar waren, setzten seit Ende der 1990er Jahre einige Staaten auf radikale Liberalisierungen. Dagegen entwickeln sich die Tschechische Republik und Slowenien zunehmend in Richtung der konservativen Wohlfahrtswelt. In Ländern wie Rumänien und Bulgarien spielen zudem die Familie und die Subsistenzwirtschaft eine tragende Rolle in der staatlich nur rudimentär ausgebauten sozialen Sicherung. Teilweise scheint eine Zuordnung zu den vier existierenden Wohlfahrtswelten möglich; insgesamt ist aber ein uneinheitlicher Hybridisierungsprozess in Mittelosteuropa feststellbar. ${ }^{28}$

26 Für einen Überblick vgl. Will Arts/John Gelissen: Three worlds of welfare capitalism or more? A state-of-theart-report, in: Journal of European Public Policy 2/2002, S. 137-158.

27 Maurizio Ferrera: The 'Southern Model' of Welfare in Social Europe, in: Journal of European Social Policy 1/1996, S. 17-37.

28 Alexandra Baum-Ceisig/Klaus Busch/Björn Hacker/Claudia Nospickel: Wohlfahrtsstaaten in Mittel- und Osteuropa. Entwicklungen, Reformen und Perspektiven im Kontext der europäischen Integration, Baden-Baden 2008. 
Annahme starker Pfadabhängigkeiten: Selbstreproduktion existierender Wohlfahrtsstaatscluster

Für Esping-Andersen ist die Entstehung differenter Wohlfahrtsstaatswelten nicht nur ein Beiprodukt unterschiedlich verlaufender Prozesse des ökonomischen, politischen und gesellschaftlichen Fortschritts. Ganz im Sinne des Historischen Institutionalismus identifiziert er nach der Herausbildung eines Wohlfahrtsstaatsregimes die Entwicklung von Pfadabhängigkeiten. Da die institutionalisierten Sozialpolitiken von Interessengruppen begleitet und verteidigt werden, hält er einen Wechsel des einmal eingeschlagenen Entwicklungspfades aufgrund der steigenden Opportunitätskosten für nahezu unmöglich. Damit aber wäre die Wohlfahrtsstaatslandschaft eingefroren und nur sehr bedingt veränderbar: Auch neu auftretende Herausforderungen, wie der gesellschaftliche Alterungsprozess und die sich erheblich wandelnden ökonomischen Rahmenbedingungen durch Globalisierung und Europäisierung, könnten die traditionellen Institutionen des Sozialstaats nicht erschüttern; die Pfadabhängigkeit wohlfahrtsstaatlicher Entwicklungen habe die Sozialpolitik vor bedeutenden Kursänderungen bewahrt. ${ }^{29}$ Einige Wissenschaftler bezweifeln die Existenz einer Vielzahl von drei oder vier Wohlfahrtsregimen und sehen lediglich zwei sich gegenüberstehende Welten der liberalen und der koordinierten Marktökonomien beziehungsweise des sozialdemokratischen Korporatismus und des Marktliberalismus. ${ }^{30}$ Doch ändert dies nichts an der postulierten Stabilität des einmal eingeschlagenen wohlfahrtskapitalistischen Pfades beziehungsweise des ,welfare state status quo“.31

Dies bedeutet für die Frage nach der Existenz eines ESM, dass dieses eine modellhafte Skizze bleibt, die den Kern der nationalen wohlfahrtsstaatlichen Arrangements in der Europäischen Union nicht berührt. Zunehmende ökonomische Interdependenzen mögen die nationalen Sozialsysteme unter Druck setzen und graduelle Anpassungen erzwingen, doch da jede Wohlfahrtsstaatswelt diesem Druck in sehr unterschiedlicher Weise ausgesetzt ist und entsprechend auch Zeitpunkt, Art und Umfang der graduellen Veränderungen variieren, bleiben die Systeme sich letztlich selbst treu. ${ }^{32}$ Esping-Andersen spricht im Hinblick auf den Umgang der Wohlfahrtsstaaten mit exogenen Schocks und neuen Risiken von einer „Selbstreproduktion“"33 der existierenden Cluster.

Dies bedeutet nicht, dass ein ESM nicht Ausdruck gemeinsamer Überzeugungen und Werte sein könnte. Es beruft sich auf gemeinsame Charakterzüge und Prinzipien und stellt somit einen unumstrittenen sozialhistorischen Acquis dar. ${ }^{34}$ Hartmut Kaelble hebt als Besonderheiten der historischen Evolution aller europäischer Wohlfahrtsstaaten die lange Geschichte sozialer Sicherung bis in die frühe Neuzeit, die weite Ausdehnung staatlicher Sozialversicherungen, einschließlich der in Europa besonders hohen Sozialausgaben und der Verankerung sozialer Grundrechte in den nationalen Verfassungen und/oder Rechtsprechungen, sowie die neu gewonnene Legitimation des Nationalstaats nach dem Zweiten Weltkrieg durch Herausbildung einer nationalen Solidargemeinschaft hervor. ${ }^{35}$ Ähnlich argumentiert

29 Paul Pierson: The new politics of the welfare state, in: World Politics 2/1996, S. 143-179; Bo Rothstein/Sven Steinmo (Hrsg.): Restructuring the welfare state. Political institutions and policy change, New York 2002.

30 Peter A. Hall/David Soskice: Varieties of capitalism. The institutional foundations of comparative advantage, Oxford 2001; Geoffrey Garrett: Partisan politics in the global economy, Cambridge 1998.

31 Gøsta Esping-Andersen: Positive-Sum Solutions in a World of Trade-Offs?, in: Gøsta Esping-Andersen (Hrsg.): Welfare states in transition. National adaptations in global economies, London 1996, S. 256-267, hier S. 267.

32 Francis G. Castles: The future of the welfare state. Crisis myths and crisis realities, Oxford 2004.

33 Esping-Andersen: Social Foundations, 1999, S. 165.

34 Kittel: EMU, EU Enlargement, and the European Social Model, 2002.

35 Hartmut Kaelble: Das europäische Sozialmodell - eine historische Perspektive, in: Hartmut Kaelble/Günther Schmid (Hrsg.): Das europäische Sozialmodell. Auf dem Weg zum transnationalen Sozialstaat, Berlin 2004, S. 31-50. 
Colin Crouch, der eine Art von Wahlverwandtschaft zwischen den westeuropäischen Staaten durch die jeweils stattfindende Ordnung, Begrenzung und Strukturierung des wirtschaftlichen Wettbewerbs, des industriellen Klassenkonflikts, politischer Interessengegensätze und kulturell-konfessioneller Spaltungen sieht. ${ }^{36}$ Ein so verstandenes, historisch gewachsenes ESM kann auch in der Gegenwart definiert werden als Verpflichtung aller EU-Staaten zu einem hohen Niveau sozialer Sicherheit, umfassenden und gesetzlich verankerten Arbeitsmarktinstitutionen sowie der institutionalisierten Fähigkeit zur konsensorientierten und demokratischen Lösung sozialer Konflikte. ${ }^{37}$ Es findet seine normative Entsprechung in den sozialen Werten und Zielen der Union in Art. 3 des Vertrags über die Europäische Union.

Dieses auf gemeinsame Werte durch ähnliche sozialpolitische Errungenschaften abstellende ESM gewinnt seine Besonderheit in Abgrenzung zu anderen Regionen der Welt: Den arbeitsrechtlichen Schutz der abhängig Beschäftigten, den bevölkerungsweiten Schutz vor extremer Armut und eine weitgehende Angleichung der Zugangschancen zu sozialen Dienstleistungen sieht Franz-Xaver Kaufmann als in allen EU-Staaten verwirklichte Aspekte, die in Abgrenzung zum US-amerikanischen, zum sozialistischen und zum ostasiatischen Umgang mit diesen Fragen, eine Art ESM konstituieren. Doch hat das ESM für Kaufmann eher eine identitätsstiftende Funktion, stellt die Suche nach ihm eine ,historische Rekonstruktion im Horizont einer gedachten Zukunft" dar. ${ }^{38}$ Eine Erosion wohlfahrtsstaatlicher Arrangements über institutionelle Reformen hinaus durch die gewachsenen globalen Interdependenzen kann er dagegen nicht erkennen; stattdessen bleibe die nationale Ebene aufgrund institutioneller Pfadabhängigkeiten weiterhin die entscheidende sozialpolitische Arena. Auch Scharpf und Vivien Schmidt stellen fest, dass Globalisierung und Europäisierung zwar viele EU-Staaten zu einer stärkeren Orientierung einzelner Politiken am Marktgeschehen veranlasst haben, doch hätten die Länder dabei weder Autorität, Legitimität noch regulatorische Kapazität so weit eingebüßt, dass es zu Auflösungs- und Konvergenzprozessen der Wohlfahrtsstaatswelten gekommen sei. ${ }^{39}$ Schärfer formuliert Jens Alber den Verbleib des ESM im Bereich der ,politischen Metaphorik“ und ,,rhetorischen Ressourcen“, 40 wenn er in empirischen Studien zu dem Schluss kommt, dass die Variationsbreite sozialer Indikatoren in der Europäischen Union sogar größer ist als der Abstand eines normativ generierten ESM zu den USA. Durch die Existenz von Pfadabhängigkeiten und das Einschlagen unterschiedlicher Lösungswege für gleiche Herausforderungen seien auch keinerlei Konvergenztrends feststellbar. ${ }^{41}$

\section{Pfadbrüche und Hybridisierung: Konvergenzprozesse auf dem Weg zum Wettbewerbsstaat}

Esping-Andersen sprach von den Niederlanden angesichts der dort stattfindenden Veränderungen des tradierten Wohlfahrtsstaatsdesigns vom „Dutch miracle“, dessen , janusköpfiges Wohlfahrtsregime" sich nicht recht in die von ihm aufgestellten Kategorien einfügen will. ${ }^{42}$ Auch jüngere Berechnungen weisen auf die schwierige Abgrenzung zwischen unter-

36 Colin Crouch: Social change in Western Europe, Oxford 1999, S. 393-408.

37 Colin Hay/Matthew Watson/Daniel Wincott: Globalisation, European Integration and the Persistence of European Social Models, POLSIS Working Paper 3/1999, S. 14.

38 Franz-Xaver Kaufmann: Nationale Traditionen der Wohlfahrtsstaatlichkeit und das „Europäische Sozialmodell“, in: Klaus Busch (Hrsg.): Wandel der Wohlfahrtsstaaten in Europa, Baden-Baden 2008, S. 17-27, hier S. 18.

39 Fritz W. Scharpf/Vivien A. Schmidt (Hrsg.): Welfare and Work in the Open Economy. From vulnerability to competitiveness, Oxford 2000.

40 Jens Alber: The European Social Model and the United States, in: European Union Politics 3/2006, S. 393-419, hier S. 414.

41 Ebenda.

42 Esping-Andersen: Social foundations, 1999, S. 88. 
schiedlichen Wohlfahrtsstaatswelten hin, decken gewichtige Veränderungen innerhalb der identifizierten Cluster auf und weisen die Existenz von Hybridfällen nach. ${ }^{43}$

Auf der Suche nach den Gründen für die teilweise erheblichen Veränderungen in einigen Wohlfahrtsstaaten muss unweigerlich das Konzept der Pfadabhängigkeit in den Fokus der Kritik geraten. Kees van Kersbergen macht gezielte Attacken konservativer und neo-liberaler Ideologen auf den tradierten europäischen Wohlfahrtsstaat seit den 1980er Jahren aus, die sich durch die Globalisierung der Ökonomie sowie durch die Binnenmarkt- und Währungsintegration in Europa noch verstärkt hätten. Sie zeigten sich in politischen Anpassungen, mit denen das Ziel der Rekommodifizierung, das heißt der Zunahme individueller Marktabhängigkeit, verbunden sei. Doch fänden diese nur selten direkt als große institutionelle Veränderungen statt, sondern durch inkrementelle Schritte, durch die Stück für Stück - einer Salamitaktik folgend - die institutionalisierten Interessen innerhalb des tradierten Wohlfahrtsstaatsdesigns ausgehöhlt werden. Dabei verändern sich die Haltungen der Akteure sowie der normative und politische Diskurs dergestalt, dass die Akkumulierung der einzelnen Leistungskürzungen in der Transformation des Wohlfahrtsstaats resultiere. ${ }^{44}$ Auch Paul Pierson blieb dieser Effekt nicht verborgen. Folglich plädiert er für eine stärkere Beachtung der zeitlichen Entwicklung und Abfolge von Veränderungsprozessen bei der Analyse des Wohlfahrtsstaats. Pfadabhängigkeit sei nie als statisches Konzept gedacht gewesen, das in einer gefrorenen Wohlfahrtslandschaft stattfindet. Gerade bei Rentenreformen in umlagefinanzierten Systemen zeige sich deutlich, wie sich heute beschlossene graduelle Leistungseinschnitte über die Zeit für künftige Generationen zu radikalen Reformen entwickeln könnten. ${ }^{45}$ Die von Pierson angeregte Betrachtung eines ,moving picture“ anstatt eines „,snapshot ${ }^{\text {“46 }}$ spiegelt sich in einem nuancierten Blick des Historischen Institutionalismus auf die Entwicklungspfade der Wohlfahrtsstaaten wider: ,Institutions matter', aber dies bedeutet nicht, dass substanzielle Veränderungen in den Politiken nicht auch ohne einen formalen Institutionenwechsel stattfinden können. Ein paradigmatischer Wandel dritter Ordnung durch Veränderung der Zielbeschreibung ${ }^{47}$ kann langfristig auch durch eine Reihe von Reformen erster und zweiter Ordnung, also durch parametrische Anpassungen beziehungsweise eine Veränderung der Instrumente, stattfinden. Pfadabweichungen, Pfadwechsel und Pfadbrüche sind denkbar. ${ }^{48}$

Besonders den konservativen und den rudimentären Wohlfahrtsstaaten werden Inflexibilitäten auf den Arbeitsmärkten sowie eine geringe Effektivität und mangelnde finanzielle Nachhaltigkeit ihrer Sozialsysteme attestiert. ${ }^{49}$ Doch auch in den skandinavischen Ländern

43 Vgl. etwa Herbert Obinger/Uwe Wagschal: Drei Welten des Wohlfahrtsstaates? Das Stratifizierungskonzept in der clusteranalytischen Überprüfung, in: Stephan Lessenich/Ilona Ostner (Hrsg.): Welten des Wohlfahrtskapitalismus. Der Sozialstaat in vergleichender Perspektive, Frankfurt Main 1998, S. 109-135; Clare Bambra: Decommodification and the worlds of welfare revisited, in: Journal of European Social Policy 1/2006, S. 73-80.

44 Kees van Kersbergen: The declining restistance of welfare states to change?, in: Stein Kuhnle (Hrsg.): Survival of the European welfare state, London 2000, S. 19-36.

45 Paul Pierson: Coping with Permanent Austerity. Welfare State Restructuring in Affluent Democracies, in: Paul Pierson (Hrsg.): The new politics of the welfare state, Oxford 2001, S. 410-456; Paul Pierson: Politics in time. History, institutions, and social analysis, Princeton 2004.

46 Paul Pierson: Increasing Returns, Path Dependence, and the Study of Politics, in: American Political Science Review 2/2000, S. 251-267, hier S. 263.

47 Peter A. Hall: Policy paradigms, social learning, and the state. The case of economic policymaking in Britain, in: Comparative Politics 3/1993, S. 275-296.

48 Wolfgang Streeck/Kathleen Thelen: Beyond continuity. Institutional change in advanced political economies, Oxford 2005; Bernhard Ebbinghaus: Can Path Dependence explain Institutional Change? Two approaches applied to Welfare State Reform, Max-Planck-Institut für Gesellschaftsforschung: MPIfG Discussion Paper 2/2005, Köln 2005.

49 André Sapir: Globalization and the Reform of European Social Models, in: Journal of Common Market Studies 2/2006, S. 369-390. 
sind in den 1990er Jahren erhebliche soziale Leistungskürzungen zu verzeichnen, durch die sich das grundsätzliche Verhältnis von Staat und Markt durch eine nun geringere Reichweite des Universalismus geändert hat.$^{50}$ Auch für Großbritannien zeigt sich, dass im Verlauf der 1980er und 1990er Jahre die auf William Beveridge zurückgehende Organisationsstruktur sozialer Sicherheit nach und nach zugunsten bedarfsorientierter Prinzipien und privater Vorsorgesysteme beschnitten wurde. ${ }^{51}$ Diese Entwicklungen sind kongruent mit Beobachtungen von Klaus Busch, der in der Nachzeichnung der Entwicklung der Sozialleistungsquote relativ zum Wirtschaftswachstum in EU-Staaten aller Wohlfahrtsstaatswelten von 1990 bis 2006 eine Reduzierung der Ausgaben für die Belange des Sozialstaats feststellt. Die über die Zeit gestreckte Analyse der institutionellen Architektur der einzelnen wohlfahrtsstaatlichen Arrangements zeigt zudem einen Prozess der zunehmenden Hybridisierung, in dem die Veränderung sozialpolitischer Institutionen durch Anleihen in verschiedenen Wohlfahrtswelten voranschreitet. ${ }^{52}$ Schon von Esping-Andersen wurde in seinen Untersuchungen ignoriert, dass Großbritannien als Paradebeispiel des liberalen Wohlfahrtsregimes im Gesundheitswesen mit seinem universellen, staatszentrierten Versorgungssystem große Gemeinsamkeiten mit sozialdemokratischen Wohlfahrtsstaaten aufweist. In ähnlicher Weise haben andere Länder einzelne Elemente verschiedener Wohlfahrtsstaatswelten in das landesspezifische Design der sozialen Sicherung integriert. Besonders auffällig ist dieser Prozess bei den zehn 2004 und 2007 neu der Europäischen Union beigetretenen mittel- und osteuropäischen Ländern.

Für die Frage nach der Existenz eines ESM hat diese Entwicklung wohlfahrtsstaatlicher Hybridmodelle in der erweiterten Europäischen Union zwei auf den ersten Blick widersprüchlich erscheinende Folgen: Einerseits nehmen die sozialen und ökonomischen Disparitäten sowie die Verteilung institutioneller Lösungsansätze innerhalb der Union durch die letzte Erweiterungsrunde zu. Einige Autoren konstatieren soziale Dumpingprozesse in der zunehmenden innereuropäischen Konkurrenzsituation und sehen eine Gefährdung des ESM, das für die ,EU-15 ' noch relativ kohärent gebildet werden konnte. ${ }^{53}$ Andererseits führt gerade die Hybridisierung des Wohlfahrtsstaats zu Konvergenzprozessen in einzelnen Sektoren der sozialen Sicherung, die ein ESM wahrscheinlicher machen. Die Beispiele für Konvergenzen der Sozialpolitiken reichen dabei von einer Einebnung des einst scharfen Gegensatzes von reinen Sozialversicherungs- und Mehrsäulenmodellen in der Altersvorsorge über zunehmende Arbeitsmarktflexibilitäten und einheitliche Ansätze in den Familien- und Bildungspolitiken bis zu gemeinsamen Strategien zur Verhinderung und Reduzierung von Armut und sozialer Ausgrenzung sowie zur gesteigerten Kosteneffizienz im Gesundheitswesen.

Die beobachteten Konvergenztrends der Wohlfahrtsstaatscluster in der Europäischen Union lassen trotz unterschiedlicher Politikmuster in der Summe mehrheitlich eine Tendenz

50 Gregg M. Olsen: Re-modelling Sweden: The Rise and Demise of the Compromise in a Global Economy, in: Julia S. O'Connor/Gregg M. Olsen (Hrsg.): Power resources theory and the welfare state. A critical approach. Toronto 1998, S. 339-368.

51 Jochen Clasen/Wim van Oorschot: Changing Principles in European Social Security, in: European Journal of Social Security 2/2002, S. 89-115, hier S. 105-115.

52 Klaus Busch: Die europäischen Perspektiven des Wohlfahrtsstaats, in: Andrea Gawrich/Wilhelm Knelangen/ Jana Windwehr (Hrsg.): Sozialer Staat - soziale Gesellschaft? Stand und Perspektiven deutscher und europäischer Wohlfahrtsstaatlichkeit, Opladen 2009, S. 253-279; Ursula Bazant/Klaus Schubert: Europäische Wohlfahrtssysteme: Vielfalt jenseits bestehender Kategorien, in: Klaus Schubert/Ursula Bazant/Simon Hegelich (Hrsg.): Europäische Wohlfahrtssysteme. Ein Handbuch, Wiesbaden 2008, S. 623-645.

53 Daniel C. Vaughan-Whitehead: L'Europe à 25. Un défi social, Paris 2005; Klaus Busch: Die Perspektiven des europäischen Sozialmodells, Hans-Böckler-Stiftung: Arbeitspapier 92, Düsseldorf 2005. 
in eine Richtung erkennen: Sozialpolitik wird zunehmend als Produktivitätsfaktor im Rahmen der europäischen Wirtschaftsverfassung verstanden. Durch inkrementelle Reformschritte, die akkumuliert die - unterschiedlich stark ausgeprägte - Rolle des Staats in der Organisation und Gewährleistung sozialer Sicherheit erheblich reduzieren, ändert sich die Gestalt der Wohlfahrtsstaaten. Diese Neuvermessung des Staat-Markt-Verhältnisses wird über die Zeit zugunsten marktkonformer Lösungen entschieden. Die tradierte Sozialgemeinschaft tritt gegenüber einem individualisierten Verständnis von Sozialschutz und der Konditionierung sozialer Rechte durch soziale Pflichten im Wohlfahrtsstaat zurück. Das Primat der Politik über das Marktgeschehen im Sinne der ,politics against markets“54 geht dabei schrittweise verloren und wird ersetzt durch eine wohlfahrtsstaatliche Politik ,,within markets". ${ }^{55}$ Das ESM definiert sich aus diesen Gegebenheiten als Produkt einer kompetitiv verstandenen Solidarität, ${ }^{56}$ als Verkörperung des gemeinsamen europäischen Übergangs vom Wohlfahrtsstaat zum Wettbewerbsstaat. ${ }^{57}$

\section{Verschränkung der Perspektiven: vier Schemata eines Europäischen Sozialmodells}

Der hier unternommene Versuch einer Strukturierung der politikwissenschaftlichen Diskursstränge zeigt, dass ein ESM zu existieren scheint, auch wenn sich je nach Perspektive signifikante Unterschiede seines Umfangs und seiner Bedeutung auftun. Damit ist das ESM mehr als eine „Chimäre“ 58 , die Begeisterung für seine Existenz beruht nicht auf einer „Selbsttäuschung“59 und seine wissenschaftliche Eingrenzung ist zwar mehrdimensional und kompliziert, doch einfacher als die ,Jagd nach einer schwarzen Katze in einem dunklen Raum". 60

Während die europäischen Integrationstheorien den Prozessverlauf auf der europäischen Ebene erklären wollen, behält die komparative Wohlfahrtsstaatsforschung die sozialpolitischen Perspektiven auf der nationalen Ebene im Blick. Aus dem Zusammentreffen der spezifischen wohlfahrtsstaats- und integrationstheoretischen Analysewege ergeben sich in einer Vier-Felder-Matrix je nach Ausprägung vier mögliche Schemata, die unterschiedliche Entwicklungsszenarien des ESM darstellen (siehe Abbildung 1):

(1) In einer Europäischen Union, in der die nationalen Wohlfahrtsstaatssysteme durch starke Pfadabhängigkeiten ihrer tradierten Sozialsysteme geprägt sind und sich die Europäisierung fast ausschließlich im Bereich der negativen Integration vollzieht, bildet sich das ESM im gemeinsamen ökonomischen Integrationsraum aus der Summe der nationalen Wohlfahrtsstaatsmodelle, die von der Integrationsdynamik weitgehend unbeeinträchtigt bleiben.

(2) In einer Europäischen Union, in der die nationalen Wohlfahrtsstaatssysteme durch starke Pfadabhängigkeiten ihrer tradierten Sozialsysteme geprägt sind und sich die Europäi-

54 Gøsta Esping-Andersen: Politics against Markets. The Social Democratic Road to Power, Princeton 1985.

55 Martin Frenzel: Verzweifelt gesucht: Ein gemeinsames Konzept für Europa. Die europäische Sozialdemokratie zwischen neoliberaler Marktanpassung und transnationaler Neubestimmung, in: vorgänge 2/2003, S. 62-71.

56 Wolfgang Streeck: Competitive Solidarity: Rethinking the "European Social Model", Max-Planck-Institut für Gesellschaftsforschung: MPIfG Working Paper 8/1999, Köln 1999.

57 Helmut Voelzkow: Governing Social Europe? Die Rolle der europäischen Sozialpolitik bei der Transformation der Wohlfahrtsstaaten in West- und Osteuropa, in: Alexandra Baum-Ceisig/Anne Faber (Hrsg.): Soziales Europa? Perspektiven des Wohlfahrtsstaates im Kontext von Europäisierung und Globalisierung, Wiesbaden 2005, S. 115-135.

58 Günther Schmid: Der Blick über den Tellerrand. Bei der Reform des Sozialstaats kann Deutschland von den sozialen Modellen seiner europäischen Nachbarn viel lernen, in: Frankfurter Rundschau, 18.06.2005.

59 „Der einzige Weg ist, dem EuGH nicht zu folgen“, Interview mit Fritz Scharpf, in: Magazin Mitbestimmung 7-8/2008, S. 18-23, hier S. 19.

60 Lamping: Auf dem Weg zu einem postnationalen Sozialstaat?, 2008, S. 611. 
sierung neben der weit vorangeschrittenen negativen, auch im Bereich der positiven Integration vollzieht, bildet das ESM im gemeinsamen ökonomischen Integrationsraum ein Dach aus gemeinsamen Werten und einzelpolitischen Vergemeinschaftungen der weiterhin relativ souveränen nationalen Wohlfahrtsstaatsmodelle aus.

(3) In einer Europäischen Union, in der sich die nationalen Wohlfahrtsstaatssysteme durch Pfadbrüche und Hybridisierungen ihrer Sozialsysteme auszeichnen und sich die Europäisierung fast ausschließlich im Bereich der negativen Integration vollzieht, ergibt sich das ESM im gemeinsamen ökonomischen Integrationsraum aus der Konkurrenz um das am besten an die Bedingungen der Wirtschaftsintegration adaptierte nationale Wohlfahrtsstaatsmodell, dem die übrigen nationalen Wohlfahrtsstaatsmodelle folgen.

\section{Abbildung 1: Das Europäische Sozialmodell aus verschiedenen wissenschaftlichen Per- spektiven}

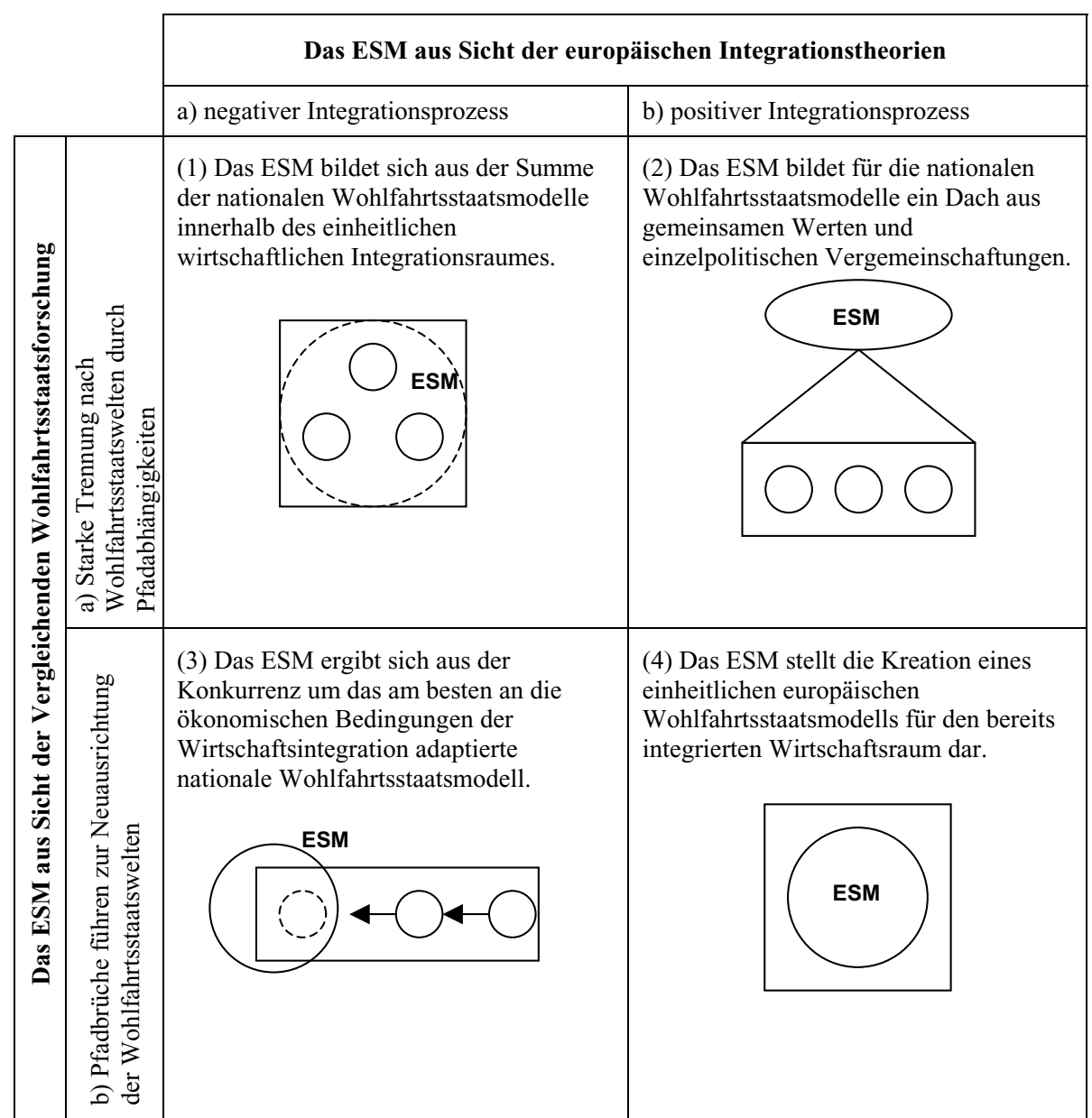

Quelle: Björn Hacker: Das liberale Europäische Sozialmodell. Rentenreformen in der EU und die Offene Methode der Koordinierung, Baden-Baden 2010, S. 60. 
(4) In einer Europäischen Union, in der sich die nationalen Wohlfahrtsstaatssysteme durch Pfadbrüche und Hybridisierungen ihrer Sozialsysteme auszeichnen und sich die Europäisierung neben der weit vorangeschrittenen negativen, auch im Bereich der positiven Integration vollzieht, stellt das ESM die Kreation eines einheitlichen Wohlfahrtsstaatsmodells im gemeinsamen ökonomischen Integrationsraum dar, mit dem die nationalen Wohlfahrtsstaatsmodelle abgelöst werden.

Diese Beschreibung ist eine theoretische Vereinfachung der oben vorgestellten Prozesse auf nationaler und europäischer Ebene. In der Realität sind die Abgrenzungen zwischen den vier schematischen Darstellungen der Generierung eines ESM natürlich mannigfaltiger, verworrener und weniger eindeutig, doch dient die vorgenommene Aufteilung einem besseren Verständnis des normativ aufgeladenen Begriffs um ein ESM.

\section{Aktuelles politisches Leitbild: Wettbewerb der Wohlfahrtsstaaten}

Doch wo befinden wir uns heute in der Integrationsentwicklung? Aus den beschriebenen Entwicklungen lässt sich ableiten, dass ein unter (1) aufgezeigtes System, in dem die Wohlfahrtsstaatswelten strikt voneinander getrennt in einem gemeinsamen Wirtschaftsraum nebeneinander bestehen, bereits der Vergangenheit angehört. Ebenso lässt sich aus den Unzulänglichkeiten der sozialen Integrationsdynamik schließen, dass ein unter (4) dargestelltes einheitliches Wohlfahrtsstaatsmodell für den gemeinsamen Integrationsraum weit entfernt ist von den realen Gegebenheiten. Je nachdem, für wie robust man die Pfadabhängigkeiten einschätzt und ob man eher die erreichten Wegmarken positiver Integrationsdynamik oder die Prädominanz der Wirtschaftsintegration betont, lässt sich das ESM heute in den Feldern (2) oder (3) verorten.

Ein genaueres Bild verspricht ein Blick auf die politisch Handelnden und die den Veränderungsdiskurs prägenden ideologischen Leitbilder, denen sie in den letzten Jahrzehnten folgten. ${ }^{61}$ Während von konservativer und liberaler Seite überall in Europa bereits seit den 1980er Jahren Angriffe auf Umfang und Reichweite staatlicher sozialer Sicherheit durchgeführt wurden, besetzte die Sozialdemokratie über lange Zeit eine Verteidigungsposition gegenüber einem Rückbau des Wohlfahrtsstaats. ${ }^{62}$ Doch als Anthony Giddens einflussreiche Idee eines „Dritten Wegs“63, den die Sozialdemokratie zwischen tradiertem Sozialstaatsverständnis und ökonomischen Ansprüchen der Globalisierung gehen sollte, sich über Tony Blairs „New Labour“ in der Europäischen Union verbreitete, veränderte sich auch das Selbstverständnis der Sozialdemokratie in erheblichem Maße. ${ }^{64}$ Anstatt weiter auf Dekommodifizierung zu setzten, verkörpert der „Dritte Weg“ ein Konzept der Rekommodifizierung. Er möchte den plötzlich als Reformblockade empfundenen, durch die Sozialdemokratie verbrieften Schutz der geerbten wohlfahrtsstaatlichen Arrangements aufgeben zugunsten einer positiven Haltung gegenüber den selbstregulatorischen Kräften der freien Marktwirtschaft. Die neo-keynesianistische Nachfrage-, Umverteilungs- und Interventionspolitik der Nachkriegsära soll eliminiert werden, um Monetarismus und angebotsorientierten Strategien

61 Renate Mayntz/Fritz W. Scharpf: Der Ansatz des akteurszentrierten Institutionalismus, in: Renate Mayntz/ Fritz W. Scharpf (Hrsg.): Gesellschaftliche Selbstregelung und politische Steuerung, Frankfurt Main 1995, S. 39-72; Vivien A. Schmidt/Claudio M. Radaelli: Policy Change and Discourse in Europe: Conceptual and Methodological Issues, in: West European Politics 2/2004, S. 183-210.

62 Mark Evans/Philip G. Cerny: "New Labour", Globalisierung und Sozialpolitik, in: Susanne Lütz/Roland Czada (Hrsg.): Wohlfahrtsstaat - Transformation und Perspektiven, Wiesbaden 2004, S. 207-229; Oliver Nachtwey: Marktsozialdemokratie. Die Transformation von SPD und Labour Party, Wiesbaden 2009.

63 Anthony Giddens: The third way. The renewal of social democracy, Cambridge 1998.

64 Aust/Leitner/Lessenich: Konjunktur und Krise des Europäischen Sozialmodells, 2002, S. 272-301; Giuliano Bonoli/Martin Powell (Hrsg.): Social Democratic Party policies in contemporary Europe, London 2004. 
Platz zu machen. Nicht den Schutz vor den Ungerechtigkeiten des Marktgeschehens, sondern die Stärkung individueller Kapazitäten und Fähigkeiten zu einer erfolgreichen, doch eigenverantwortlichen Teilnahme hieran soll der vorsorgende Sozialstaat der Zukunft ermöglichen.

Vor dem Hintergrund dieser Zielbeschreibung wird die Entwicklung eines ESM als „System von Wettbewerbsstaaten" 65 verständlich. Giddens teilt die Meinung, dass in der Europäischen Union aus ehemals stark pfadabhängigen Wohlfahrtsstaaten über die Zeit Hybridmodelle entstanden sind. Nachdem die von ihm entwickelte Strategie des „Dritten Wegs“ maßgeblich dazu beigetragen hat, Veränderungen auf der nationalen Ebene in Richtung einer stärkeren Marktdominanz in allen EU-Staaten voranzutreiben, entwickelt er auf dieser Grundlage seine Vision eines, neuen ESM‘. Dazu gehören unter anderem eine Verpflichtung der Mitgliedstaaten zur verantwortlichen Haushaltspolitik, die Schaffung von Arbeitsplätzen als wichtigste Strategie des Sozialschutzes, die Stärkung der Arbeitsmarktflexibilität, keine übermäßige Belastung von Vermögenden durch hohe Steuern und Abgaben, eine Umschichtung finanzieller Mittel von Alten zu Jungen und die Verlängerung der Lebensarbeitszeit, die Förderung von Teilzeitarbeit und gezielte Aktivierungsprogramme für einzelne Bevölkerungsgruppen im Sinne der ,employability“ 66

Die Europäische Kommission unter ihrem Präsidenten Jacques Delors sah bis Mitte der 1990er Jahre die Verwirklichung des ESM mit einem stärkeren Ausbau der sozialen Dimension, mit sozialpolitischer Koordinierung und sozioökonomischer Angleichung der Mitgliedstaaten verbunden. Die beiden zentralen Integrationsprojekte Binnenmarkt und Währungsunion sollten flankiert und eingebettet werden in die Verpflichtung zum gemeinsamen sozialen Fortschritt. ${ }^{67}$ Mit dieser Sichtweise bricht das neue politische Leitbild des Wettbewerbsgedankens. Beeinflusst hiervon wurden nahezu alle politischen Kräfte und auch die europäischen Institutionen. Die Lissabon-Strategie, die Sozialagenda und der Entwurf eines Vertrags über eine Verfassung für Europa legen Zeugnis ab von einem eindimensionalen Verständnis der Europäischen Union als ökonomischen Zweckverbund, in dem die (sozial-)politische Integration als nachrangig betrachtet wird. ${ }^{68}$ Am herrschenden Paradigma einer Wirtschafts- und Wettbewerbsunion hat auch die Finanz- und Wirtschaftskrise der letzten Jahre nichts verändert. Im Gegenteil deuten die implementierten und diskutierten Krisenlösungsmechanismen auf eine Verschärfung des Wettbewerbsansatzes in der Europäischen Union hin. ${ }^{69}$ Das ESM ergibt sich hierin als Konkurrenzprodukt national gestalteter Wohlfahrtsstaatsmodelle, die zu einem (wenn auch zentralen) Wettbewerbsfaktor unter vielen degradiert werden. Dabei trifft die Auflösung starrer Pfadabhängigkeiten auf ein durch Marktkonkurrenz dominiertes Integrationsverständnis. Dasjenige nationale Wohlfahrts-

65 Busch: Die Perspektiven des europäischen Sozialmodells, 2005.

66 Anthony Giddens: Die Zukunft des Europäischen Sozialmodells, Friedrich-Ebert-Stiftung: Internationale Politikanalyse 3/2006.

67 Vgl. etwa Europäische Kommission: Europäische Sozialpolitik. Ein zukunftsweisender Weg für die Union, Weißbuch, KOM (94) 333.

68 Vgl. etwa Höpner/Schäfer: Grenzen der Integration, 2010, S. 3-20; Armin Schäfer: Verfassung und Wohlfahrtsstaat: Sozialpolitische Dilemmas Europäischer Integration, in: Internationale Politik und Gesellschaft 4/2005, S. 120-141; Jenny Preunkert: Chancen für ein soziales Europa? Die Offene Methode der Koordinierung als neue Regulierungsform, Wiesbaden 2009, hier S. 289-298; Kenneth A. Armstrong/Iain Begg/Jonathan Zeitlin: JCMS Symposium: EU Governance after Lisbon, in: Journal of Common Market Studies 2/2008, S. 413-450.

69 Hans-Jürgen Bieling: Eine gemeinsame europäische Krisenüberwindungsstrategie - Probleme und Perspektiven, in: Gudrun Hentges/Hans-Wolfgang Platzer (Hrsg.): Europa - quo vadis? Ausgewählte Problemfelder der europäischen Integrationspolitik, Wiesbaden 2011, S. 69-92; Björn Hacker/Till van Treeck: Wie einflussreich wird die europäische Governance? Reformierter Stabilitäts- und Wachstumspakt, Europa 2020-Strategie und „Europäisches Semester“, Friedrich-Ebert-Stiftung: Internationale Politikanalyse 11/2010. 
staatsmodell wird als ESM ausgezeichnet, das am meisten Arbeitsplätze schafft und seine Strukturen sozialer Sicherheit soweit zurückbaut, dass das Marktgeschehen nur minimal beeinträchtigt wird. Voluntaristische Integrationselemente, wie beispielsweise die OMK, dienen in diesem ESM in erster Linie dazu, das am besten an die Marktintegration adaptierte Wohlfahrtsstaatsmodell zu identifizieren. ${ }^{70}$ Damit befinden wir uns derzeit im oben beschriebenen Feld (3) der ,wettbewerbsgetriebenen Harmonisierung“" ${ }^{71}$ an deren Ende ein , liberales ESM`stehen wird, falls es nicht zu einer Reduzierung des Drucks der negativen Integrationsdynamik und zu einem Kurswechsel hin zu einer stärker marktkorrigierenden und marktgestaltenden Funktion europäischer Politiken im Sinne eines neu zu begründenden ,solidarischen ESM‘ kommt.

\section{Studies on the European Union}

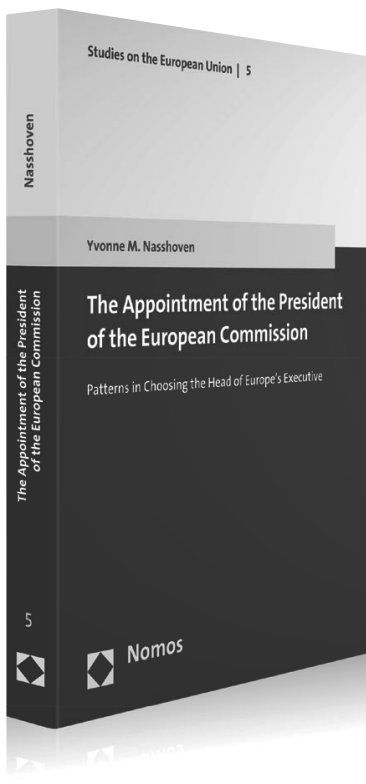

\section{The Appointment of the President of the European Commission}

Patterns in Choosing the Head

of Europe's Executive

Von Yvonne M. Nasshoven

2011, Band 5, 260 S., brosch., 39,- $€$

ISBN 978-3-8329-6446-7

Der vorliegende Band analysiert die Auswahl politischen Führungspersonals am Beispiel des Präsidenten der Europäischen Kommission. Hierzu werden Muster im Ernennungsprozess identifiziert und in den jeweiligen politischen Kontext gesetzt.

Bitte bestellen Sie im Buchhandel oder versandkostenfrei unter $\bullet$ www.nomos-shop.de

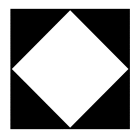

Nomos

70 Björn Hacker: Das liberale Europäische Sozialmodell, 2010.

71 Hans-Jürgen Urban: Europäisierung der Gesundheitspolitik? Zur Evolution eines Politikfeldes im europäischen Mehrebenen-System, Wissenschaftszentrum Berlin (WZB) für Sozialforschung, Berlin 2003, S. 50. 